\title{
Numerical Analysis of Turbulent Combustion in a Model Swirl Gas Turbine Combustor
}

\author{
Ali Cemal Benim, ${ }^{1}$ Sohail Iqbal, ${ }^{1}$ Franz Joos, ${ }^{2}$ and Alexander Wiedermann ${ }^{3}$ \\ ${ }^{1}$ Center of Flow Simulation (CFS), Department of Mechanical and Process Engineering, \\ Düsseldorf University of Applied Sciences, Josef-Gockeln-Str. 9, 40474 Düsseldorf, Germany \\ ${ }^{2}$ Laboratory of Turbomachinery, Helmut Schmidt University, Holstenhofweg 85, 22008 Hamburg, Germany \\ ${ }^{3}$ Engineering Gas Turbines, MAN Diesel and Turbo SE, Steinbrinkstr. 1, 46145 Oberhausen, Germany \\ Correspondence should be addressed to Ali Cemal Benim; alicemal.benim@hs-duesseldorf.de
}

Received 30 November 2015; Revised 28 July 2016; Accepted 17 August 2016

Academic Editor: Hong G. Im

Copyright (C) 2016 Ali Cemal Benim et al. This is an open access article distributed under the Creative Commons Attribution License, which permits unrestricted use, distribution, and reproduction in any medium, provided the original work is properly cited.

\begin{abstract}
Turbulent reacting flows in a generic swirl gas turbine combustor are investigated numerically. Turbulence is modelled by a URANS formulation in combination with the SST turbulence model, as the basic modelling approach. For comparison, URANS is applied also in combination with the RSM turbulence model to one of the investigated cases. For this case, LES is also used for turbulence modelling. For modelling turbulence-chemistry interaction, a laminar flamelet model is used, which is based on the mixture fraction and the reaction progress variable. This model is implemented in the open source CFD code OpenFOAM, which has been used as the basis for the present investigation. For validation purposes, predictions are compared with the measurements for a natural gas flame with external flue gas recirculation. A good agreement with the experimental data is observed. Subsequently, the numerical study is extended to syngas, for comparing its combustion behavior with that of natural gas. Here, the analysis is carried out for cases without external flue gas recirculation. The computational model is observed to provide a fair prediction of the experimental data and predict the increased flashback propensity of syngas.
\end{abstract}

\section{Introduction}

Modern gas turbines are to provide high efficiency, reliability, and stability, while meeting strict low emission requirements, with emerging additional requirements such as fuel flexibility. In that respect, the combustor is obviously a core component, and a detailed understanding of the complex flow, heat, and mass transfer processes in the flame is of great importance. Experimental investigation of gas turbine combustion is difficult and can provide only limited information due to practical limitations. Numerical simulations can provide detailed insight and reduce the number of costly experiments. Nevertheless, the highly complex processes in the combustor are difficult to model and the simulations are afflicted with inaccuracies. Thus, development of mathematical and numerical models for GTC and their experimental validation have been a continuous endeavor, to which the present work is aimed to provide a contribution.
Fureby [1] applied an EDC-type combustion model in combination with LES to analyze GTC. Lörstad et al. [2] analyzed the reacting flow in the Siemens SGT-800 burner experimentally and numerically applying RANS and LES approaches, along with an EDC-type combustion model, a focus of the work being on the effect of burner fuel distribution on flame dynamics. A recent study on URANS and LES modelling of GTC for a Siemens scaled combustor was presented by Goldin et al. [3] using the flamelet generated manifold model as combustion model in combination with LES and turbulent flame speed models in combination with URANS. They found that LES predictions of the mean and RMS axial velocity, mixture fraction, and temperature fields do not show an improvement over the RANS. Again recently, ALSTOM's reheat combustor was successfully analyzed by Kulkarni et al. [4] applying a novel combustion model based on a composite reaction progress variable, along with a tabulated chemistry approach and the stochastic fields turbulence-chemistry interaction model. 
In the simulation of GTC, one of the main challenges is turbulence modelling. This is caused by the highly nonisotropic turbulence structure, which is created by the high swirl levels applied to induce a flame-stabilizing vortex breakdown. In the previous work of present authors [57], it was shown that a three-dimensional and unsteady formulation that can resolve the unsteadiness of coherent structures is necessary for achieving sufficient accuracy in such flows (as also confirmed by the recent work of other authors [1-4]). This requirement is fulfilled (at different levels) by URANS and LES approaches. In the present application, a URANS formulation is used, as the basic modelling approach, in combination with a two-equation turbulence model. In modelling turbulent swirling flows, the RSM has generally been found to be more accurate compared to twoequation turbulence models (while convergence difficulties may impede its application in some cases). We have found, however, that this superiority of the RSM is given basically for the RANS, that is, steady-state formulations. In an unsteady formulation such as URANS, we quite often observed that two-equation models perform similarly well. In this case, the use of a two-equation turbulence model is reasonable, since the additional computational overhead of the RSM (5 additional transport equations and less superior convergence behavior) does not seem to be justified. Still, for comparison, URANS is applied also in combination with the RSM to one of the investigated cases. For this case, LES is also used for turbulence modelling.

Modelling of the turbulence-chemistry interaction is the further main challenge, of course. A method, which is found to be adequate in modelling turbulence-chemistry interaction is the EDC [8], as successfully applied to GTC by several authors [1]. A drawback of EDC is, however, that an individual transport equation needs to be solved for each species, which increases the computational demand proportionally with the considered number of species. For meeting the current demands of combustion technology, reaction mechanisms with always increasing level of sophistication are required that incorporate a rather large number of species. In combination with computationally demanding turbulence modelling approaches such as URANS and LES, which are necessary for achieving sufficient accuracy as discussed above, the computational costs become extremely high, especially for real applications in the industrial development environment. On the other hand, the laminar flamelet method (LFM) [9] provides a very efficient way of considering detailed reaction kinetics in turbulent combustion, where a completely detailed reaction mechanism can be incorporated via a few variables that describe flamelet characteristics. Although the validity of the LFM for GTC was questioned in the past, based on Damköhler number arguments, it was shown, later on $[10,11]$, that purely dimensional arguments neglecting the transient effects may be misleading and typical GTC operation conditions are rather in the validity range of the LFM. Thus, the LFM is used as the turbulent combustion model in the present study. It is argued that a mixture fraction-reaction progress variable characterization of the flamelets is convenient for the present partially premixed flame, as will be described in more detail below.

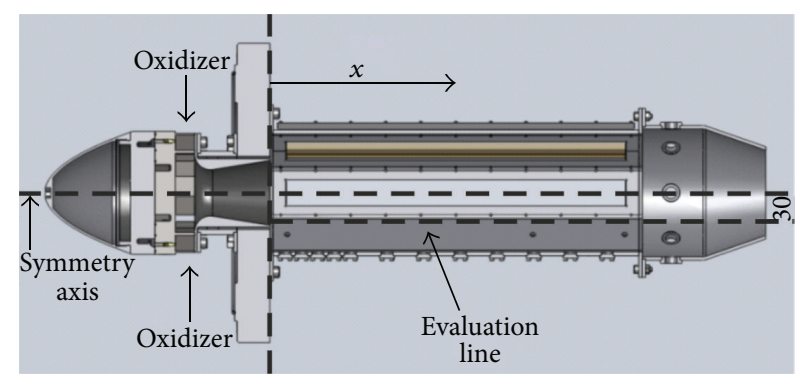

FIgURE 1: Combustor geometry.

The computational model is applied to predict turbulent combustion in a model gas turbine combustor firing natural gas (NG) and syngas (SG). First, a validation study is performed for the NG flame with flue gas recirculation (FGR). Numerical results are compared with the experimental data, where a reasonably fair agreement is observed. Then, the validated model is applied to predict the combustion behavior of SG, in comparison to NG, without FGR.

The motivation of the present work has been the assessment of the performance of the advocated laminar flamelet method in predicting gas turbine swirl flames considering the realistic fuel injection configuration of an industrial swirl burner. Moreover, the assessment of the predictive capability of the approach for the $\mathrm{CO}$ and $\mathrm{NO}$ emissions under flue gas recirculation and using syngas as fuel (instead of natural gas) has been a further motivation. The coherent consideration of the all abovementioned aspects on the same rig and within the same modelling framework is a novelty of the present investigation.

\section{The Test Case}

Experiments were performed at the Turbomachinery Laboratory of Helmut Schmidt University, Hamburg. The CAD drawing of the atmospheric model combustor, which is equipped by a single swirl burner with 12 channels, is illustrated in Figure 1. The oxidizer stream passes through a hood (not displayed in the figure) and enters radially into the channels of the radial swirler.

Fuel is injected into the cross-flowing air by 12 injection holes, each located at the wall of each swirler channel. After passing through the swirler channels, each of which having an inclination of $45^{\circ}$, the fuel-air mixture enters the central converging-diverging burner nozzle and, subsequently, into the octagonal main combustion chamber. A converging exhaust gas nozzle is attached to the combustor exit to avoid reverse flow. Based on the local unburnt bulk axial velocity and the diameter at the exit of the converging-diverging burner nozzle, the Reynolds number turns out to be about 34,000 . Assuming a perfect guidance by the swirler channels and conservation of angular momentum within the burner, and defining the swirl number as the ratio of the maximum swirl velocity to the bulk axial velocity, a swirl number of about 0.9 can be estimated at the burner nozzle outlet. A 
TABLE 1: Composition (vol, \%) of fuel stream.

\begin{tabular}{lccccccc}
\hline & $\mathrm{CH}_{4}$ & $\mathrm{C}_{3} \mathrm{H}_{8}$ & $\mathrm{CO}_{2}$ & $\mathrm{CO}$ & $\mathrm{H}_{2}$ & $\mathrm{O}_{2}$ & $\mathrm{~N}_{2}$ \\
\hline NG & 92.5 & 5.2 & 1.3 & - & - & - & 1 \\
SG & 10 & 22 & 4 & 22 & 40 & - & 2 \\
\hline
\end{tabular}

TABLE 2: Composition (vol, \%) of oxidizer stream.

\begin{tabular}{lccccc}
\hline & $\mathrm{O}_{2}$ & $\mathrm{H}_{2} \mathrm{O}$ & $\mathrm{CO}_{2}$ & $\mathrm{Ar}$ & $\mathrm{N}_{2}$ \\
\hline FGR0 & 20.6 & 1 & - & 0.9 & 77.5 \\
FGR20 & 17.9 & 2.3 & 2.2 & 0.9 & 76.7 \\
\hline
\end{tabular}

detailed description of the setup and measurement methods are provided in [12].

The combustor was designed for premixed/partially premixed operation. In the experiments [12], different fuel compositions were considered. The compositions of the NG and SG that are considered in the present work are presented in Table 1.

The measurements [12] were performed for investigating the effect of external flue gas recirculation (FGR). Thus, different oxidizer compositions were investigated [12]. The oxidizer compositions that are considered in the present study are shown in Table 2 (FGR0 corresponding to "zero" flue gas ratio, i.e., to pure air).

In the present analysis, totally three cases are analyzed: NG with FGR (NG-FG20), NG without FGR (NG-FGR0), and SG without FGR (SG-FGR0). For the NG-FG20 case, the mixture composition was adjusted to have an adiabatic flame temperature of about $1525 \mathrm{~K}$ (corresponding to an equivalence ratio of about 0.5 , fuel and oxidizer mass flow rates being $0.0009217 \mathrm{~kg} / \mathrm{s}$ and $0.03575 \mathrm{~kg} / \mathrm{s}$, and combustor inlet temperature $573 \mathrm{~K}$ ). For SG-FGR0 case, an operation point was chosen corresponding to a slightly lower adiabatic temperature of $1450 \mathrm{~K}$.

\section{Modelling}

3.1. Grid, Boundary Conditions, and Outline of the Mathematical Model. A block structured mesh, consisting of 1.2 million cells, is used. A detailed view of the surface mesh is illustrated in Figure 2. At the fuel and oxidizer inlets, constant profiles are prescribed for velocities, temperature, and mass fractions that result from the global combustor data. At the outlet, zerogradient boundary conditions are applied for convectivediffusively transported variables, along with a constant static pressure. The no-slip walls are assumed to be adiabatic. Near solid walls, the turbulence modelling is augmented by the use of standard, logarithmic wall-functions. The near-wall grid resolution was such that the $y^{+}$values [13] were not exceeding 120 and had an average value of approx. 50 in the burner. Although the applied near-wall resolution is not very fine (to resolve the wall boundary layers), we assume that this would not lead to a serious deterioration of the predictive capability, since the governing processes of the problem such as jet mixing or vortex breakdown are of rather free-shear layer type and, thus, not much affected by the wall boundary layers.

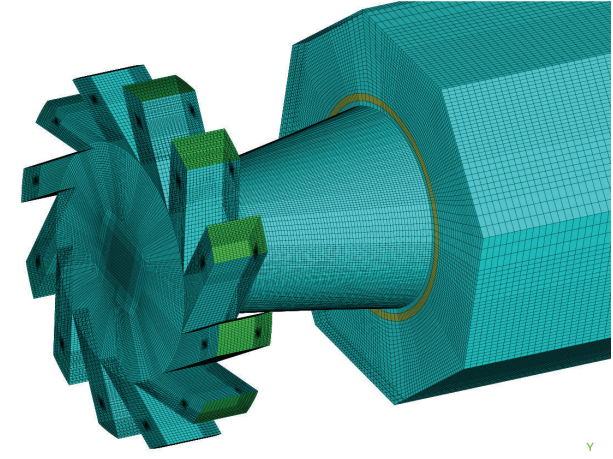

Figure 2: The mesh (detail view of surface mesh).

For the computational investigation, the finite volume method based open source CFD code OpenFOAM [13] is used, where a pressure-correction scheme (PIMPLE, which is a combination of PISO and SIMPLE schemes) was applied for treating the velocity-pressure coupling. As turbulence model, the Shear Stress Transport (SST) model [13] is applied within a URANS context as the main approach. For scalars, a gradient-diffusion approximation is used, along with the assumption of constant turbulent Schmidt numbers of value 0.7. For comparison, the Reynolds Stress Model (RSM) [14] is also used in comparison with URANS, for one of the cases. Additionally, the Large Eddy Simulation (LES) [15] approach is also employed as turbulence modelling strategy for this case, using the Smagorinsky-Lilly subgrid-scale model [15].

A second-order upwind scheme was applied to discretize the convective terms in the transport equations for all the variables. A first-order Euler scheme was used for time stepping, since stability problems were quite often encountered with a second-order time discretization. The time step size is chosen in such a way that cell Courant numbers do not exceed unity. Starting from an initial field, the numerical simulations were performed for a time period, which is long enough to allow the development of a quasi-periodic flow field that is no more dependent on the initial conditions. After this state, the time-averaging of the results was started, which was continued until the time-averaged fields did not show any substantial change in time.

Along with the three momentum equations, the pressurecorrection equation, and two equations for the turbulence model, three additional differential transport equations (four equations, if $\mathrm{NO}$ is included) are solved for combustion modelling which are discussed in the following section.

3.2. The Combustion Model. The turbulence-chemistry interactions necessitate the use of a combustion model, if flow turbulence is not directly simulated but modelled. In the present work, the laminar flamelet method (LFM) is used. According to the usual assumptions of the LFM [9], for a steady, one-dimensional, adiabatic, laminar diffusion flame, all thermochemical dependent variables $(\varphi)$ can be expressed as unique functions of the mixture fraction $Z$ and the stoichiometric value of the scalar dissipation rate $\chi_{\mathrm{st}}$ as

$$
\varphi=\varphi\left(Z, \chi_{\mathrm{st}}\right) \text {. }
$$


The mixture fraction $Z$ represents the mass fraction of fuel stream locally in the unburnt mixture. For $\chi_{\mathrm{st}}$, an assumed functional dependence on $Z$ (e.g., as the one suggested by Peters [9]) is quite commonly used. Such functional relationships (see (1)) are obtained by performing $1 \mathrm{D}$ laminar flame calculations, once for all, and made available in tabulated form (flamelet libraries) for the subsequent CFD analysis. Note that the laminar flame extinguishes for a sufficiently large value of the scalar dissipation rate. This is the socalled "quenching limit." For a quenched laminar flame, relationships of type (1) describe the nonburning state. In the turbulent flow, the average values of the thermochemical dependent variables such as the species mass fractions and the static mixture temperature can be obtained by the following expression, utilizing presumed PDFs (the expression is written for a Favre-averaged quantity but holds analogously for a Reynolds-averaged quantity).

$$
\widetilde{\varphi}=\int_{0}^{\infty} \int_{0}^{1} \varphi\left(Z, \chi_{\mathrm{st}}\right) P(Z) P\left(\chi_{\mathrm{st}}\right) d Z d \chi_{\mathrm{st}} .
$$

In the above expression, a statistical independence of $Z$ and $\chi_{\mathrm{st}}$ is assumed along with the assumption of a singledelta PDF for $\chi_{\text {st }}$. The presumed PDF for $Z$ is typically controlled by its two moments, that is, the average value $\widetilde{Z}$ and the variance $\widetilde{Z^{\prime \prime 2}}$. The modelled time-averaged differential transport equations of these variables [16] are

$$
\begin{aligned}
& \frac{\partial \bar{\rho} \widetilde{Z}}{\partial t}+\frac{\partial\left(\bar{\rho} \widetilde{u}_{j} \widetilde{Z}\right)}{\partial x_{j}}-\frac{\partial}{\partial x_{j}}\left[\left(\frac{\mu}{\sigma}+\frac{\mu_{t}}{\sigma_{t}}\right) \frac{\partial \widetilde{Z}}{\partial x_{j}}\right]=0, \\
& \frac{\partial \bar{\rho} \widetilde{Z^{\prime \prime 2}}}{\partial t}+\frac{\partial\left(\bar{\rho} \widetilde{u}_{j} \widetilde{Z^{\prime \prime 2}}\right)}{\partial x_{j}}-\frac{\partial}{\partial x_{j}}\left[\left(\frac{\mu}{\sigma}+\frac{\mu_{t}}{\sigma_{t}}\right) \frac{\partial \widetilde{Z^{\prime \prime 2}}}{\partial x_{j}}\right] \\
& =2 \frac{\mu_{t}}{\sigma_{t}} \frac{\partial \widetilde{Z}}{\partial x_{j}} \frac{\partial \widetilde{Z}}{\partial x_{j}}-c \bar{\varrho} \frac{\epsilon}{k} \widetilde{Z^{\prime \prime 2}},
\end{aligned}
$$

where $c$ is a model constant ( $c=2.0$ is used). The "steady" LFM outlined above is known to perform successfully for a large class of nonpremixed flames. However, it is known [9] that it cannot accurately describe phenomena such as local extinction, reignition, and flame lift-off. Thus, it is principally not adequate for premixed/partially premixed flames like the present one.

Therefore, in the present study, a flamelet model based on the mixture fraction and the reaction progress variable $(C)$ is adopted, which is more suitable for premixed/partially premixed flames. The model was originally proposed by Pierce and Moin [17] within an LES framework, who suggested a parameterization of the flamelets based on the reaction progress variable instead of the scalar dissipation rate. The complete locus of solutions of the flamelet equations results in a so called S-shaped curve, an example of which is shown in Figure 3, for the NG-FGR20 case. The upper branch represents the stable burning flamelets till the turning point, which corresponds to the quenching limit. After the quenching limit the curve continues to decrease scalar dissipation rate and describes the unstable flamelets whereas the lower branch

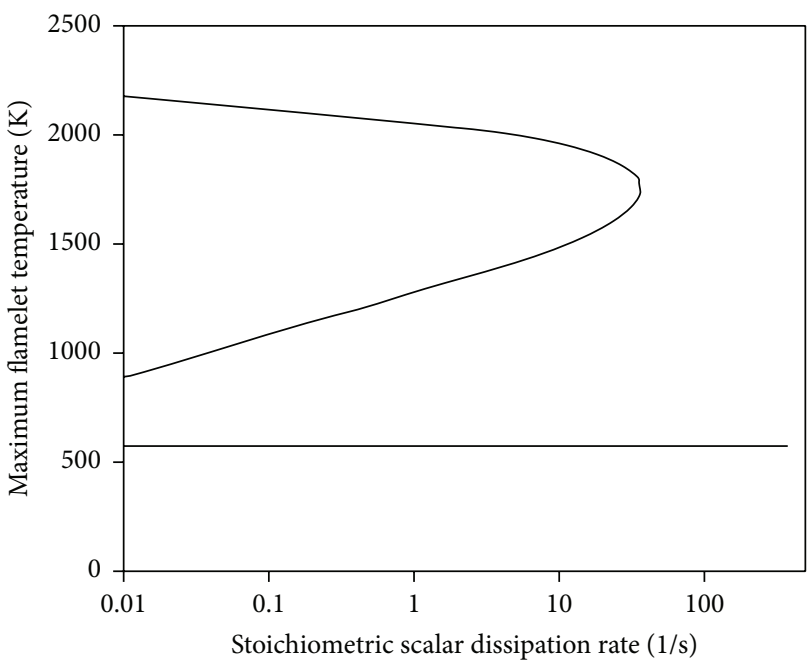

FIGURE 3: S-shaped curve for the NG-FGR20 flame.

corresponds to nonburning flamelets. Pierce and Moin [17] parameterized the flamelets based on the so-called reaction progress variable by projecting the flame states horizontally along the S-shaped curve (Figure 3).

The reaction progress variable can be defined in different ways. In the present work, a temperature-based definition is preferred (the alternative species-based definitions are rather difficult to handle in the present case with FGR due to the existence of combustion products at the oxidizer inlet)

$$
C=\frac{T_{\max , \chi}-T_{u}}{T_{b}-T_{u}} .
$$

In (5), $T_{\max , x}$ is the maximum static temperature locally prevailing within the stretched $1 \mathrm{D}$ laminar flame. $T_{u}$ and $T_{b}$ denote the unburnt and burnt equilibrium temperatures of the mixture. Please note that $T_{\max , \chi}$ also depends on the scalar dissipation rate, incorporating its effect indirectly. Thus, within this concept, the laminar flamelet functional relationships are established as

$$
\varphi=\varphi(Z, C) .
$$

Assuming $Z$ and $C$ are independent, with the help of presumed PDFs, the average values of the thermochemical variables are obtained in a similar fashion to (2). In the present work, a single-delta PDF is assumed for $C$ (as it was commonly assumed in the previous applications of the model including the original work of Pierce and Moin [17]). For $Z$, a beta PDF $(\beta)$ is used. Thus, the average values of the static mixture temperature and all species mass fractions (except that of NO) are obtained from

$$
\widetilde{\varphi}=\int_{0}^{1} \varphi(Z, \widetilde{C}) \beta\left(Z ; \widetilde{Z}, \widetilde{Z^{\prime \prime 2}}\right) d Z .
$$

What remains is the field information on the averaged progress variable to close the system. For this, a differential 
transport equation is solved, which is derived following Bray's approximation [18] of the chemical source term reading as

$$
\frac{\partial \bar{\rho} \widetilde{C}}{\partial t}+\frac{\partial\left(\bar{\rho} \widetilde{u}_{j} \widetilde{C}\right)}{\partial x_{j}}-\frac{\partial}{\partial x_{j}}\left[\left(\frac{\mu}{\sigma}+\frac{\mu_{t}}{\sigma_{t}}\right) \frac{\partial \widetilde{C}}{\partial x_{j}}\right]=\overline{\theta_{C}}
$$

In (8) $\theta_{C}$ is defined as

$$
\theta_{C}=\frac{Q}{c_{P}\left(T_{b}-T_{u}\right)}
$$

where $Q$ and $c_{P}$ denote volumetric heat release rate and the mean isobaric heat capacity, respectively. The volumetric heat release rate $Q$ is obtained from the $1 D$ laminar flame libraries.

Since NO reactions are very slow compared to the main combustion reactions, the extraction of NO mass fractions out of the flamelet data (see (6)) is inappropriate. Thus for NO, an additional transport equation is solved. This approach does not create a serious conflict with the detailed combustion mechanism underlying the LFM, due to the extremely small NO mass fractions having negligibly small effects on the transport processes and heat release in the flame. The modelled NO transport equation reads as

$$
\begin{aligned}
& \frac{\partial \bar{\rho} \widetilde{Y_{\mathrm{NO}}}}{\partial t}+\frac{\partial\left(\bar{\rho} \widetilde{u}_{j} \widetilde{Y_{\mathrm{NO}}}\right)}{\partial x_{j}}-\frac{\partial}{\partial x_{j}}\left[\left(\frac{\mu}{\sigma}+\frac{\mu_{t}}{\sigma_{t}}\right) \frac{\partial \widetilde{Y_{\mathrm{NO}}}}{\partial x_{j}}\right] \\
& =\overline{S_{\mathrm{NO}}} .
\end{aligned}
$$

The critical issue here is the determination of the source term $\left(S_{\mathrm{NO}}\right)$. As previously demonstrated [11], a quite effective means for this purpose is the extraction of its instantaneous value out of the flamelet libraries, like many other thermochemical variables (via (6)) and obtaining the time-averaged value (for closing (10)) by means of PDFs (see (7)). Since the source term stems from a detailed reaction mechanism, all NO formation paths are simultaneously considered, whereas the thermal NO formation is expected to be the dominating one in the present application.

The flamelet libraries (see (6)) are constructed by 1D steady, adiabatic laminar flame calculations using the FlameMaster code [19], before the field calculations of the turbulent reacting flow by means of CFD. As the underlying reaction mechanism, the GRI Mech 3.0 [20] is used, assuming a Lewis number of unity for all species. Within the subsequent CFD calculations, the local values of the Favre-averaged species mass fractions and temperature are obtained from (7), which relies on the solution of three differential transport equations for $\widetilde{Z}$ (see (3)), $\widetilde{Z^{\prime \prime 2}}$ (see (4)), and $\widetilde{C}$ (see (8)). If NO prediction is required, $(10)$ is additionally solved for $\widetilde{Y_{\mathrm{NO}}}$. The above-described combustion model is implemented in OpenFOAM [13].

3.3. On the Adequacy of the Grid Resolution. A formal grid independence study was not performed. The grid is constructed based on our previous experience on similar flames $[6,7]$. The following analysis of the turbulent scales of the present results indicates that the applied grid resolution is reasonably fine, based on the suggestions of Celik et al. [21] and our previous experience [6,7]. Within the framework of LES, different measures were proposed for assessing the grid resolution, such as the Grid Index (GI) defined as ratio of the local grid size (assumed to be given by the third root of the cell volume) to the Kolmogorov length scale

$$
\mathrm{GI}=\frac{\Delta}{l_{K}},
$$

with

$$
l_{K}=\left(\frac{v^{3}}{\varepsilon}\right)^{1 / 4}
$$

According to Celik et al. [21], GI should be smaller than 25, for achieving sufficient accuracy for LES. In our previous LES modelling work [7] on swirling flows, we found that the results obtained for GI $\leq 50$ do not remarkably differ from those obtained for GI $\leq 25$. This led to the conclusion that the criterion GI $\leq 50$ was sufficient for good accuracy, at least for the present class of flow problems. In the present work, besides the URANS approach as the main turbulence modelling strategy, LES is also used. We assume that the LES grid resolution criteria can be regarded to be useful for URANS too, since URANS principally has less stringent grid resolution requirements compared to LES. Figure 4 presents the distribution of GI (see (11)) in a plane through the swirler (at the channel mid-height) and in a plane through the middle of the combustor, at a time step (for the NG-FGR20 flame).

One can see that there are large regions fulfilling GI $<25$. In the remaining regions, the values remain mostly within the band $25<\mathrm{GI}<50$. Thus, the calculated GI values (Figure 4 ) can be interpreted to indicate a sufficiently fine grid for the present purposes.

\section{Results and Discussion}

The results will be presented in two parts; in the first part, the results for the NG flame with FGR (NG-FGR20) will be discussed. In the second part, the results for the NG and SG flames without FGR (NG-FGR0, SG-FGR0) will be presented.

4.1. NG Flame with FGR. The present combustor is designed to operate in premixed/partially premixed mode [12]. The fuel jets mix into the oxidizer in the swirler channels and then along the converging-diverging burner nozzle (Figure 1). Given the high swirl level of the flow, the sudden area expansion at the exit of the burner nozzle induces a vortex breakdown, that is, an inner recirculation zone, which shall act as an aerodynamic flame holder at which the flame front shall be anchored. Depending on the degree of mixing achieved in the burner, a premixed or partially premixed flame can result.

Although it was obvious that the LFM based on the mixture fraction and the scalar dissipation rate (see (1)-(4)) is not convenient for the present case, it was still applied to see its performance in the specific application and to demonstrate 


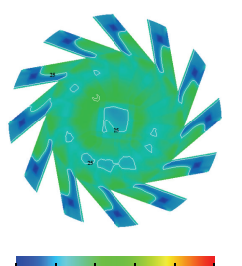

$0 \quad 20 \quad 40 \quad 6080100$ GI

(a)

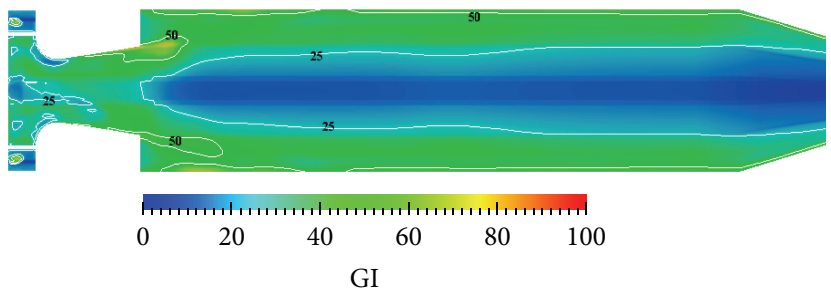

(b)

FIGURE 4: Distribution of GI in planes through (a) swirler and (b) combustor.

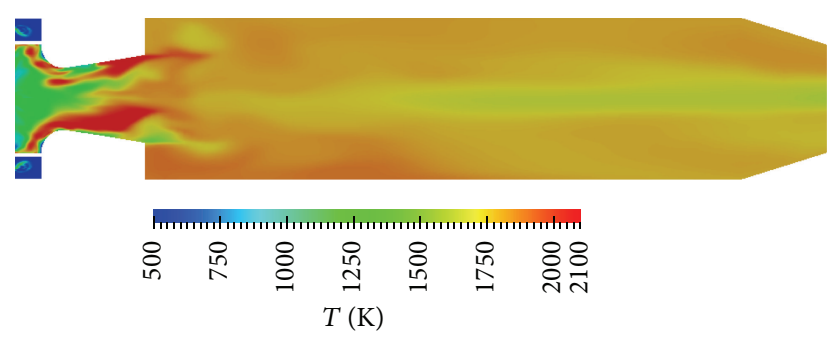

Figure 5: Predicted temperature field at a time step in a plane through combustor, calculated using mixture fraction-scalar dissipation rate based LFM (see (1)-(4)) for NG-FGR20 flame.

the improvement of the method by the introduction of the reaction progress variable (see (3)-(9)). Figure 5 displays the predicted temperature field at a time step in a plane through the combustor, which was calculated using the mixture fraction-scalar dissipation rate based LFM (see (1)(4)), for the NG-FGR20 flame. The predicted flame burns in diffusion mode (Figure 5), and a flame lift-off could not be predicted. The rather low temperatures $(<1000 \mathrm{~K})$ in the swirler channels are caused by locally high strain rates and quenching effects in the close vicinity of fuel injection holes. Downstream the swirler channels, where the local strain rate is lower, locally near-stoichiometric combustion leads to high flame temperatures about $2100 \mathrm{~K}$.

In the present work, the LFM based on the mixture fraction and the reaction progress variable (see (5)-(9)) is used as the combustion model. In the following, the results obtained by this model will be presented.

Distributions of the axial velocity predicted by the mixture fraction-reaction progress variable LFM for the NGFGR20 flame, at two different time steps, in a plane through the combustor are presented in Figure 6, using URANSSST, URANS-RSM, and LES approaches. The predicted inner recirculation zone due to vortex breakdown can be seen in Figure 6, which exhibits a quite unsteady and three-dimensional structure. As expected, LES (Figure 6(c)) resolves finer structures compared to URANS (Figures 6(a) and 6(b)), whereas URANS-RSM (Figure 6(b)) also seems to capture finer structures than URANS-SST (Figure 6(a)). It is interesting to note that the recirculation zone is attached to the burner back plate. In the present plots in a twodimensional plane (Figure 6), the extension of the threedimensional recirculation zone to the burner back plate

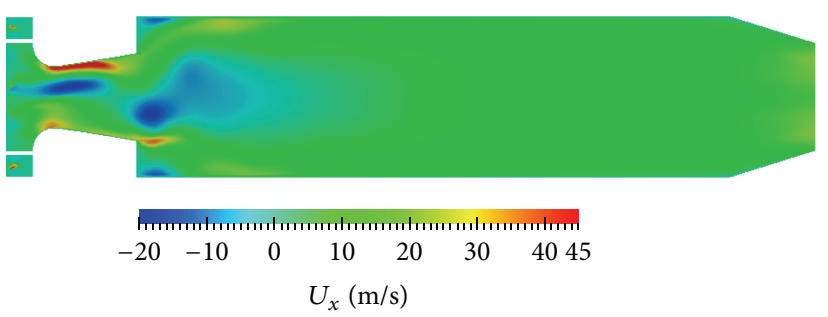

(a)

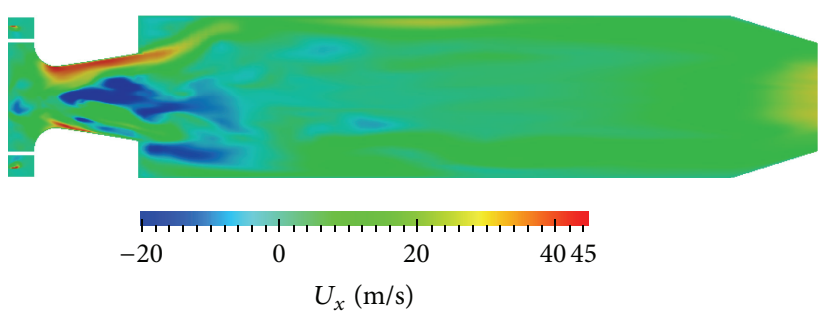

(b)

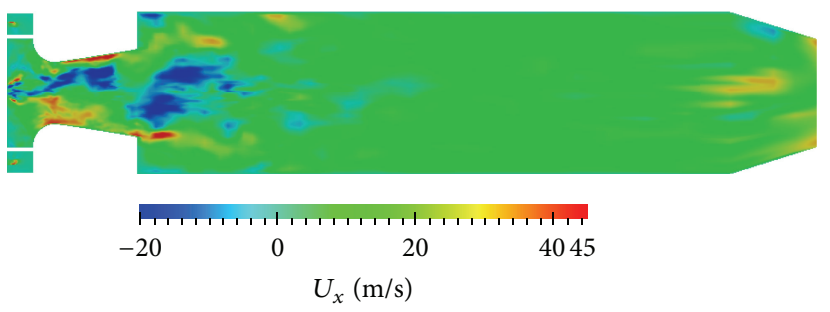

(c)

FIgURE 6: Predicted fields of axial velocity at an arbitrary time step in a plane through combustor by the mixture fraction-reaction progress variable based LFM for NG-FGR20 flame: (a) URANS-SST, (b) URANS-RSM, and (c) LES.

cannot directly be seen but deduced, for example, from Figure 6(a).

Time-averaged predictions of the axial velocity component and the velocity magnitude, as predicted by URANSSST, URANS-RSM, and LES, for the NG-FGR20 flame, are shown in a plane through the combustor in Figure 7. One can see that the time-averaged axial velocity field exhibits a bubble-shaped vortex breakdown recirculation zone (Figures $7(\mathrm{a}), 7(\mathrm{c})$, and $7(\mathrm{~d})$ ). The slender negative axial velocity regions that were precessing inside the burner (Figure 6(a)) disappear in the time averaging for URANS-SST 


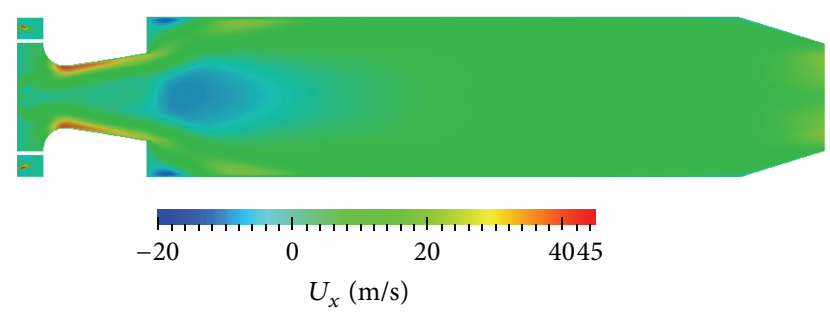

(a)

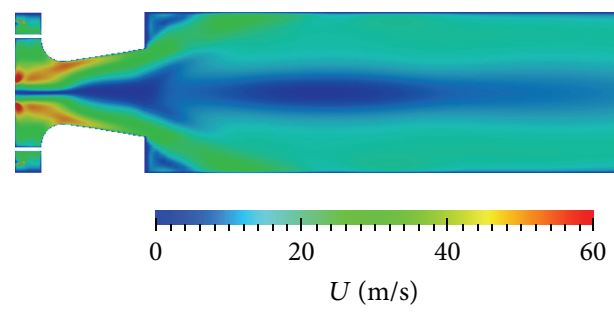

(b)

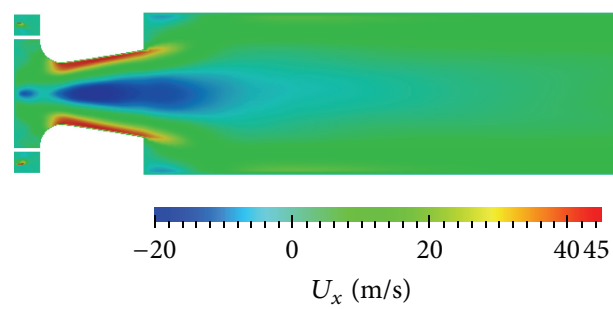

(c)

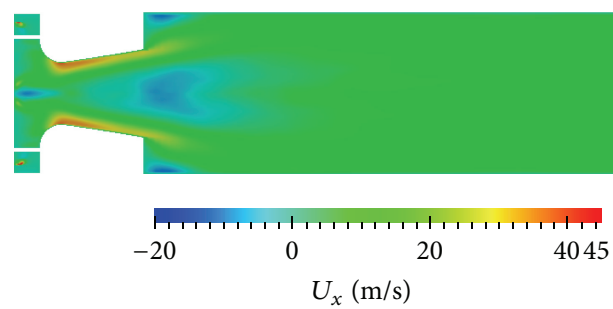

(d)

FIgURE 7: Predicted time-averaged velocity fields in a plane through combustor for NG-FGR20 flame: (a) axial velocity URANS-SST, (b) velocity magnitude URANS-SST, (c) axial velocity URANS-RSM, and (d) axial velocity LES.

(Figure 7(a)). URANS-RSM predicts a quite intensive inner recirculation zone with higher negative axial velocities and a deeper extension of the time-averaged recirculation zone into the burner, practically up to the burner back plate (Figure $7(\mathrm{c})$ ). The velocity magnitude plot (Figure $7(\mathrm{~b})$ ) indicates the very strong vortex core in the burner, which expands downstream and extends along the combustor length.

As an indication of the flow turbulence, the distribution of the representative RMS value of the velocity fluctuations $\left(U_{\text {rms }}\right)$ normalized by a reference velocity $\left(U_{\text {ref }}\right)$ is presented in Figure 8, for a plane through the combustor. The LES results are used for this purpose. The representative $U_{\text {rms }}$ is obtained from the calculated turbulence kinetic energy $(k)$ from $U_{\mathrm{rms}}=\sqrt{(2 / 3) k}$. Doing so, the turbulence kinetic energy $k$ is calculated from $k=\left(\overline{u^{\prime 2}}+\overline{v^{\prime 2}}+\overline{w^{\prime 2}}\right)$, where $\overline{u^{\prime 2}}, \overline{v^{\prime 2}}$,

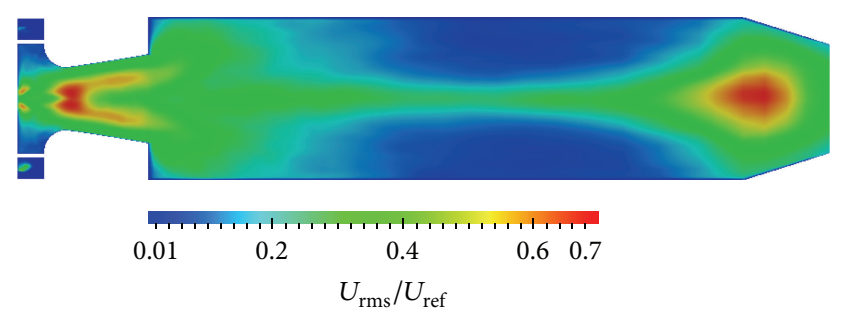

Figure 8: Predicted $U_{\text {rms }} / U_{\text {ref }}$ in a plane through the combustor (LES).

and $\overline{w^{\prime 2}}$ represent the RMS of the three velocity components resolved by LES. As the reference velocity, the bulk axial velocity at the throat of the burner nozzle is used $\left(U_{\text {ref }}=\right.$ $23 \mathrm{~m} / \mathrm{s}$ ). One can see that quite high turbulence intensities prevail especially in the burner nozzle and in its downstream, where the vortex breakdown occurs.

The predicted time-averaged fields of temperature and CO mass fraction for the NG-FGR20 flame resulting from URANS-SST, URANS-RSM, and LES calculations are displayed in Figure 9. As it can be deduced from the distributions given in Figure 9, the predictions indicate that the flame is now anchored at the burner nozzle exit, as confirmed by the experiments, and no high temperature zones exist near burner walls (Figure 5), which is important for the integrity of the hardware. One can also observe that URANS-SST (Figure 9(a)) and LES (Figure 9(b)) predict a flame front, which is practically positioned just downstream the burner nozzle, whereas URANS-RSM indicates a more strongly inclined flame front, reaching deeper into the burner on its axis (Figure 9(c)). LES (Figure 9(d)) predicts a slightly thicker flame brush than the both URANS solutions (Figures 9(a) and $9(\mathrm{c})$ ). The combustion takes place in the premixed mode, where a maximum temperature of about $1500 \mathrm{~K}$ is achieved behind the curved flame brush (Figures 9(a), 9(c), and 9(d)). The CO mass fraction quickly attains a local maximum in the reaction zone (Figure 9(b)) and gets depleted downstream.

The predicted and measured profiles of time-averaged temperature along the "combustor axis" and along the "evaluation line" (Figure 1) are compared in Figure 10. Note that the displayed domain in Figure 10 covers the combustor domain downstream the burner nozzle exit $(x>0$, Figure 1$)$.

Both experiments and predictions show (Figure 10) a quite rapid increase of the temperature across the relatively thin flame front, as already indicated by the temperature plot presented in Figure 9. The evaluation line touches the edge of the burner nozzle exit at $x=0$ (Figure 1). Since the flame front is curved and rather thick near the edge of the burner nozzle exit, especially for URANS-SST and LES (Figure 9), the evaluation line (Figure 1) crosses through the whole reaction zone. On the combustor axis, the displayed part in Figure 10(a) $(x \geq 0)$ covers the rather thin reaction zone of URANS-SST and LES only partially (only the rear part, since the flame brush is located slightly within the burner nozzle). The reaction zone of URANS-RSM is not covered at all on the combustor axis shown in Figure 10(a), since the flame is located at a substantially upstream position compared to $x=0$. Thus, the temperature increase observed 

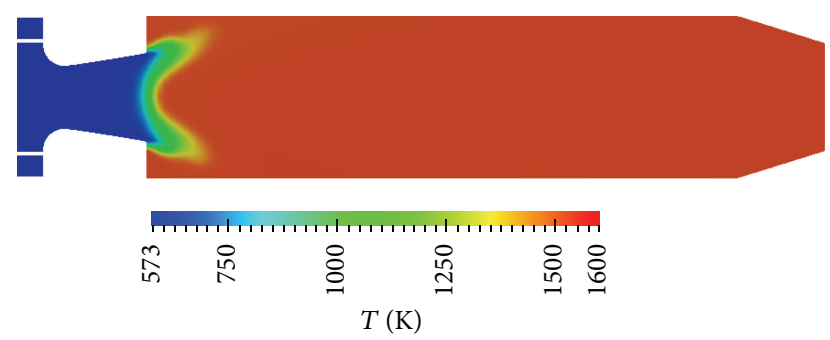

(a)
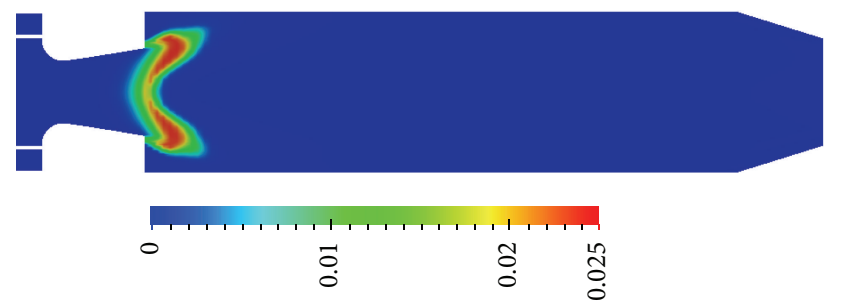

Mass fraction $\mathrm{CO}(-)$

(b)
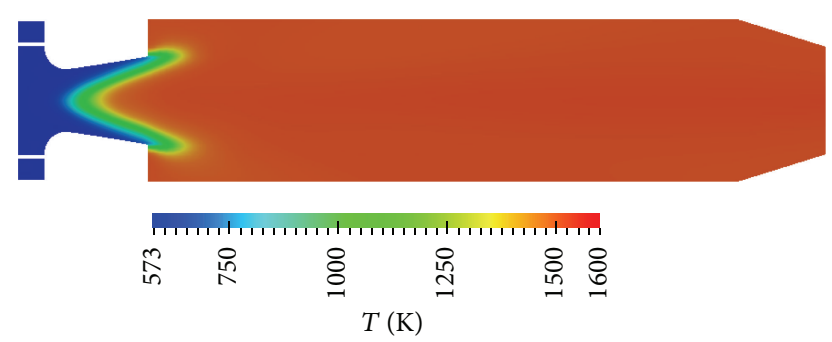

(c)
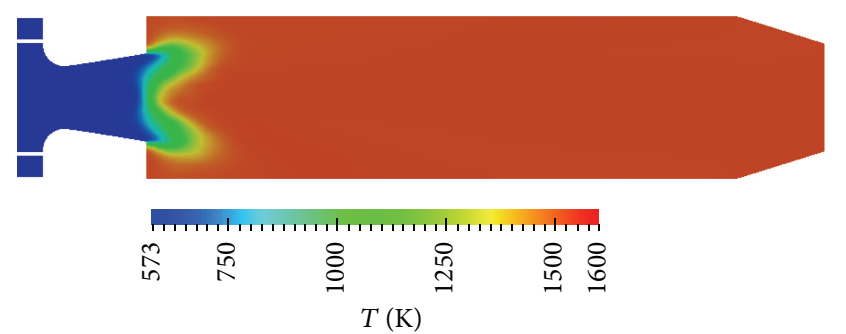

(d)

Figure 9: Predicted time-averaged fields of (a) temperature URANS-SST, (b) CO mass fraction URANS-SST, (c) temperature URANS-RSM, and (d) temperature LES, in a plane through combustor.

along the evaluation line (Figure 10(b)) is larger and takes place in a larger distance compared to the temperature rise along the combustor axis (Figure 10(a)). This trend is qualitatively the same in all predictions as well as in the experiments. On the evaluation line, which encompasses the whole flame front, the measured temperatures exhibit a local maximum (Figure 10(b)). Calculations agree quite well with the measurements in predicting this temperature peak in the flame zone (Figure 10(b)). This is predicted slightly better by LES compared to the other models. URANS-SST predictions are quite close to those of LES. The temperature gradient predicted by URANS-RSM occurs at a slightly upstream position compared to the experiments and other predictions
(Figure 10(b)), due to the difference in the predicted flame front shape as discussed above. Similar trends are generally observed for the combustor axis (Figure 10(a)). Due to the quite upstream position of the flame front predicted by URANS-RSM on the combustor axis, as discussed above, the URANS-RSM results do not exhibit a temperature gradient for the profile shown in Figure 10(a), for the combustor axis. Downstream the initial peak, the measured temperatures decline, whereas the predicted values do not decrease. The burnt gas temperature is overpredicted by about $100-150 \mathrm{~K}$ (Figure 10). This may be due to the assumption of no heat loss to the environment (adiabatic combustor walls) in the predictions.

The predicted and measured profiles of time-averaged CO mass fraction along the combustor axis and along the "evaluation line" (Figure 1) are compared in Figure 11. The evaluation line displayed in Figure 11(b) cuts through the whole flame front (as discussed in the preceding paragraph). Thus, peak values produced in the flame zone are well captured. One can see in Figure 11(b) that quite large CO mass fractions are predicted by all models at the beginning of the evaluation line, which agree quite well with the measurements. LES seems to predict even higher maximum values compared to URANS-SST and URANS-RSM (Figure 11(b)). Downstream of this peak, the measurements show a quite sharp decay. The calculations agree well with this gradient only for a short distance (for $x \leq$ approx. $0.05 \mathrm{~m}$ ). Beyond this region, the measured values continue to decay sharply up to approx. $x=$ $0.1 \mathrm{~m}$, where the predictions exhibit a rather milder decay, which may be due to an underprediction of the CO burnout rate in this region (Figure 11(b)). This results in a local overprediction of the $\mathrm{CO}$ mass fractions beyond the initial region $(x \geq 0.05 \mathrm{~m})$ (Figure 11(b)). Compared to the other models, the decay of the CO mass fractions is predicted at best by URANS-RSM, which, however, overpredicts the exit value (Figure 11(b)). URANS-SST and LES agree better with the experiments at the exit (Figure 11(b)). For the combustor axis (Figure 11(a)), the predicted peak values occur at a more upstream position compared to Figure 11(b), due to the curved shape of the flame front. Due to similar effects, an overprediction of the $\mathrm{CO}$ mass fractions for a region along the centreline is observed (Figure 11(a)). The predicted combustor outlet values are quite close to the measurements (Figure 11(a)). For the evaluation line (Figure 11(a)) and especially for the combustor axis (Figure 11(a)), the shape of the experimental curve is predicted at best by URANSRSM. In comparison, LES generally predicts a quite gradual decay (instead of an initial sharp decay followed by a rather flat curve, as observed in experiments), whereas URANSSST results may be seen to be qualitatively between those of URANS-RSM and LES (Figure 11).

The predicted and measured profiles of time-averaged NO mass fraction along the combustor axis and along the "evaluation line" (Figure 1) are compared in Figure 12. For NO mass fraction, the calculations overpredict the experiments throughout (Figure 12). However, the discrepancy is not too large, and this is at least partially due to the overpredicted temperature field (Figure 10). The relative performance of the different models correlates with the temperature predictions. 


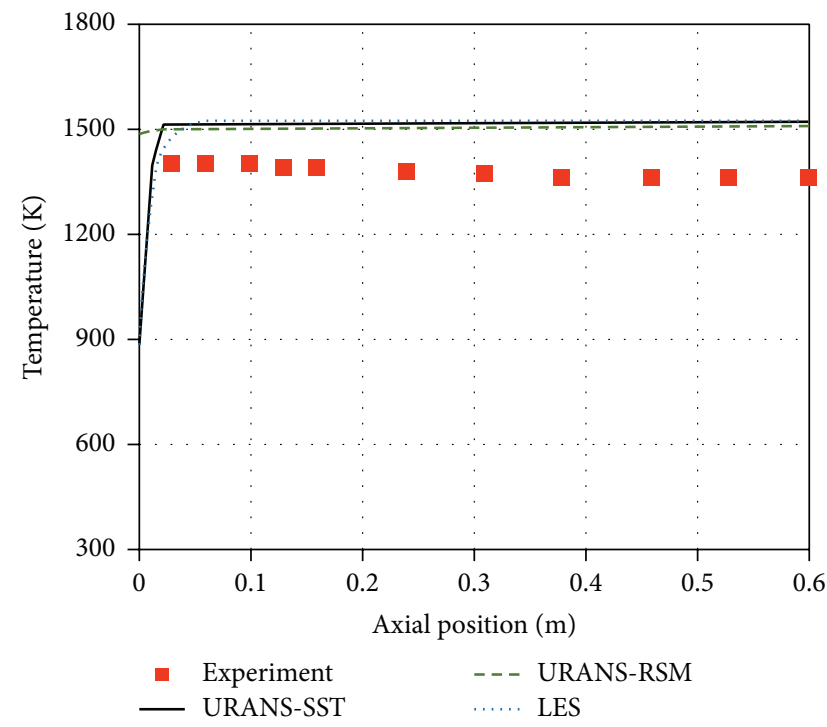

(a)

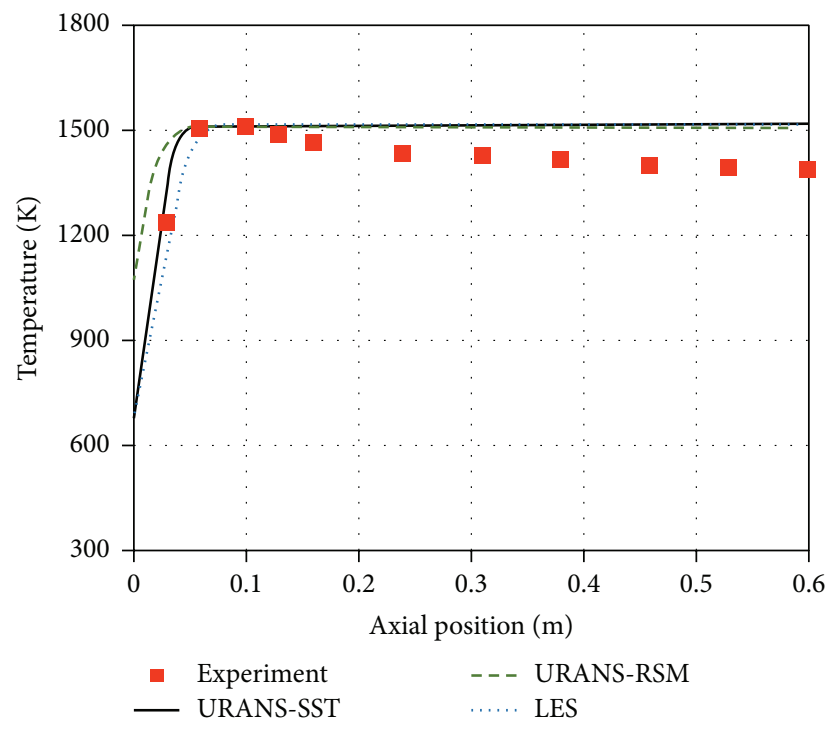

(b)

FIGURE 10: Predicted and measured profiles of time-averaged temperature along (a) combustor axis and (b) evaluation line for NG-FGR20 flame.

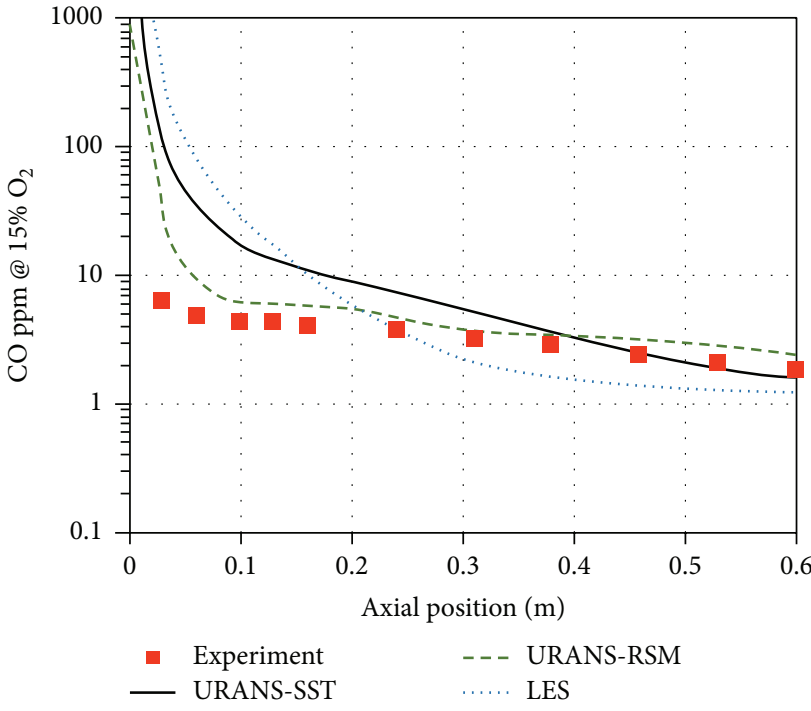

(a)

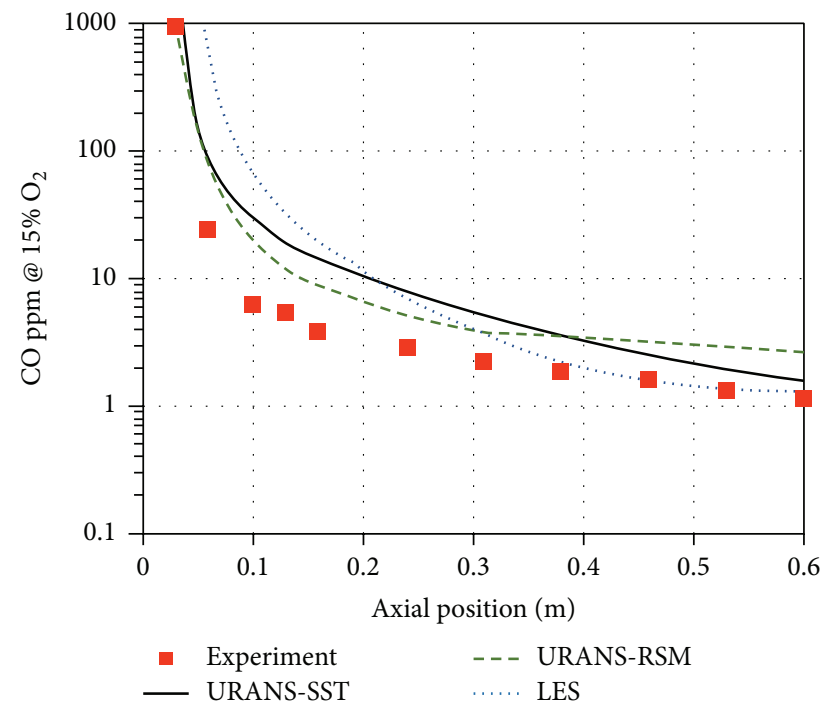

(b)

FIGURE 11: Predicted and measured profiles of time-averaged CO mass fraction along (a) combustor axis and (b) evaluation line for NG-FGR20 flame.

LES seems to perform slightly better than the other models in the initial parts, where the NO mass fractions sharply increase. URANS-SST also shows a rather good agreement there. The URANS-RSM predictions show a slight upstream shift compared to the other results, since the flame front was predicted at a slightly upstream position in comparison (Figure 9). Overall, a fair agreement between the predictions and the measurements is observed (Figure 12).

4.2. NG and SG Flames without FGR. All results presented in this section are obtained by URANS-SST. The predicted time-averaged temperature fields for the NG-FGR0 and SGFGR0 flames are displayed in Figure 13.

As seen in Figure 13, the NG-FGR0 case exhibits a shorter, less curved flame brush (Figure 13(a)) compared to NGFGR20 (Figure 9(a)). In the central part of the burner, the flame front shows a slightly higher penetration into the burner nozzle for NG-FGR0 (Figure 13(a)) compared to NGFGR20 (Figure 9(a)). However, there is no "flashback" as the burner walls are not exposed to high temperatures. On the contrary, in the SG-FGR0 flame, although the adiabatic flame temperature is lower, a flashback tendency is predicted 


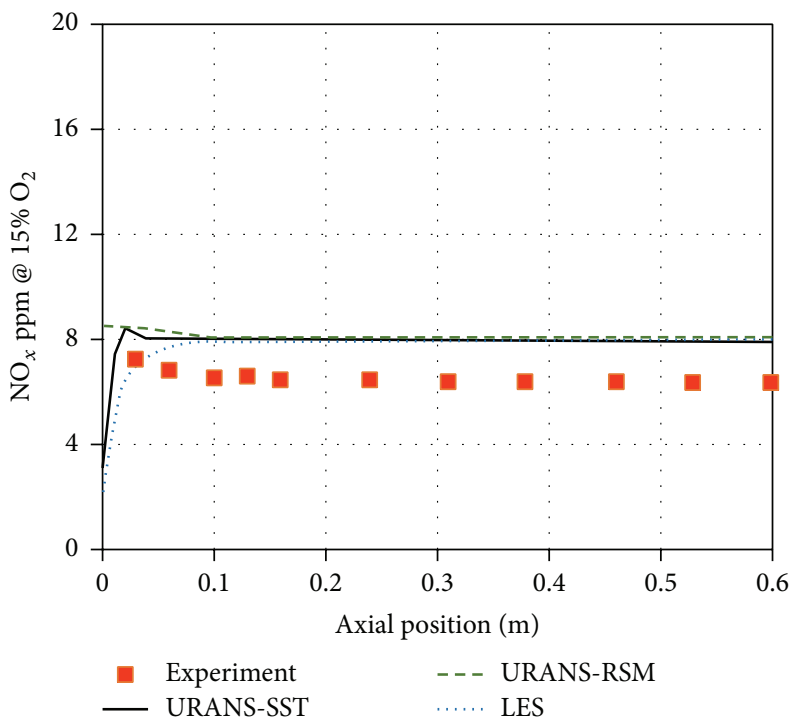

(a)

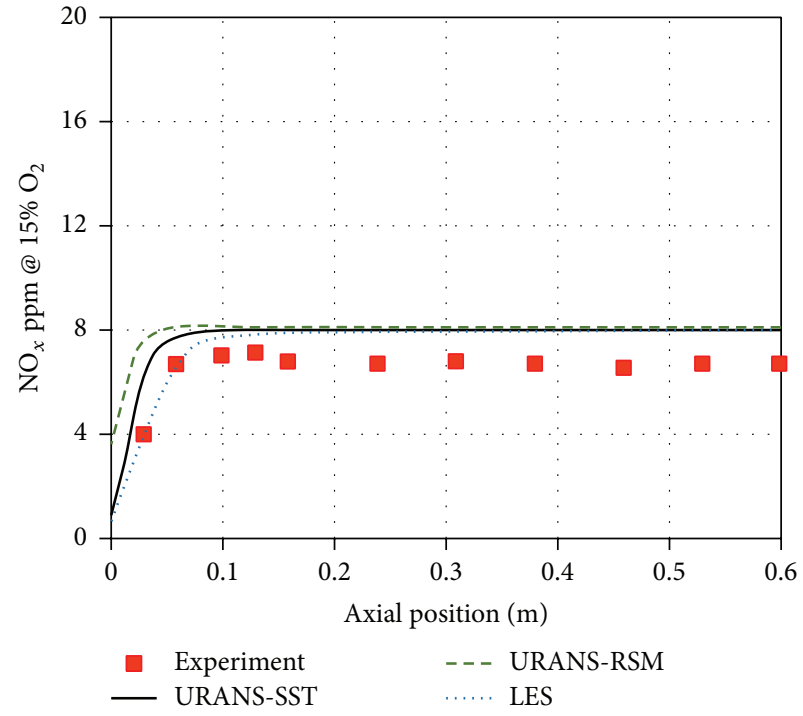

(b)

Figure 12: Predicted and measured profiles of time-averaged NO mass fraction along (a) combustor axis and (b) evaluation line for NGFGR20 flame.

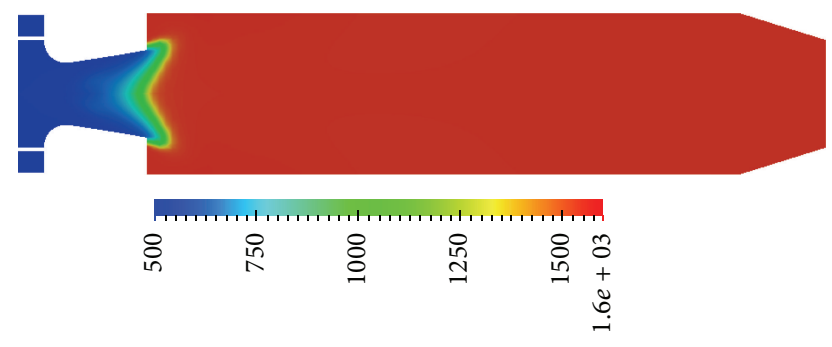

$T(\mathrm{~K})$

(a)

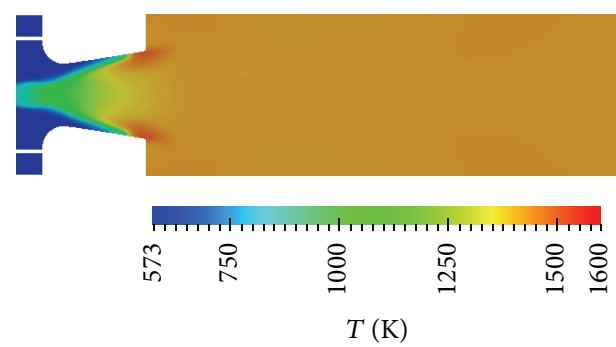

(b)

FIGURE 13: Predicted time-averaged temperature fields in a plane through combustor for (a) NG-FGR0 flame and (b) SG-FGR0 flame.

(Figure 13(b)) as the flame propagates deep upstream into the burner with an accompanying overheating of the burner walls around the edges of the nozzle exit. There are several mechanisms that can trigger a flashback [22]. Flashback due to combustion induced vortex breakdown is less likely to be the cause, since the expansion ratio, which is observed to correlate to this phenomenon [22], is even smaller for the SG (where the flashback occurs) compared the NG flame. Thus, it likely that the flashback is due to flame propagation in
TABLE 3: Combustor exit CO and NO mass fractions for NG-FGR0.

\begin{tabular}{lcc}
\hline & Measured & Predicted \\
\hline CO ppm @ 15\% O $\mathrm{O}_{2}$ & 0.12 & 3.42 \\
$\mathrm{NO}$ ppm @ 15\% O $\mathrm{O}_{2}$ & 3.02 & 7.95 \\
\hline
\end{tabular}

the core or in the boundary layers due to increased laminar flame speed by the higher hydrogen content of SG. The test rig was not designed to make detailed measurements in the burner. Thus, it is not clear how well the predicted flashback tendency corresponds to the experiments, for this operation point. However, although not quantified and documented, a generally strong flashback propensity of the used SG fuel was observed during the experimental investigation [12].

Figure 14 shows the contours of the time-averaged mixture fraction with respect to the flame front for both (NGFGR0 and SG-FGR0) flames. The flame front is characterized by the $\widetilde{C}=0.5$ isoline of the reaction progress variable, which is indicated by the thick red line in the figure. Thinner black lines indicate the isolines of the mixture fraction, where the corresponding $\widetilde{Z}$ values are typed nearby. The fully premixed values of $\widetilde{Z}$ are 0.025 and 0.065 for the NG-FGR0 and SGFGR0 flames, respectively. One can see that the NG-FGR0 flame burns in fully premixed regime (similar to NG-FGR20). For the SG-FGR0 flame, the mixture fraction varies within the range about $0.035-0.075$ indicating a partially premixed combustion (Figure 14).

For NG-FGR0 and SG-FGR0 cases, measurements were not available within the combustor but at the combustor outlet. Tables 3 and 4 compare the predicted and measured $\mathrm{CO}$ and NO mass fractions at the combustor outlet, for NGFGR0 and SG-FGR0 flames, respectively.

For CO, the prediction for the SG-FGR0 flame is quite close to the measurement (Table 3). For the NG-FGR0 flame, 


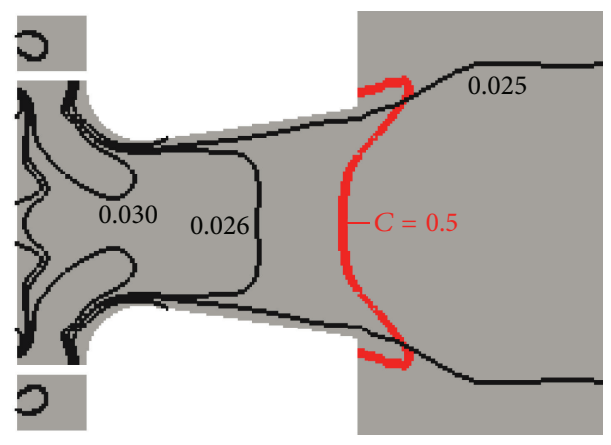

(a)

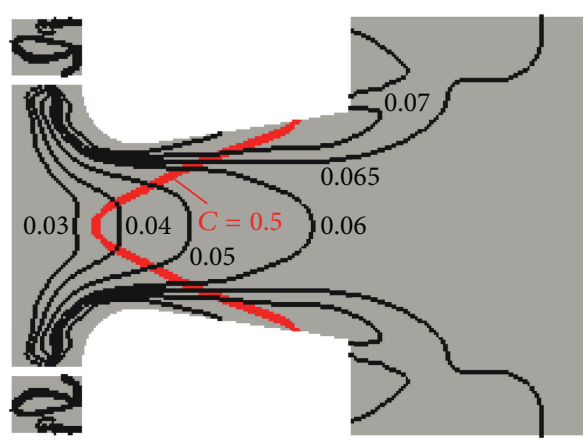

(b)

FIGURE 14: Predicted time-averaged isolines of the mixture fraction with respect to the time-averaged flame front $(\widetilde{C}=0.5)$ for (a) NG-FGR0 flame and (b) SG-FGR0 flame (the premixed $\widetilde{Z}$ values are 0.025 and 0.065 for NG-FGR0 and SG-FGR0 flames, resp.).

TABLE 4: Combustor exit CO and NO mass fractions for SG-FGR0.

\begin{tabular}{lcc}
\hline & Measured & Predicted \\
\hline CO ppm @ 15\% O $\mathrm{O}_{2}$ & 1.04 & 1.74 \\
NO ppm @ 15\% O $\mathrm{O}_{2}$ & 0.41 & 2.61 \\
\hline
\end{tabular}

the deviation between the prediction and measurement is larger but still not too large (Table 4). For NO, there is a moderate overprediction for both flames (Tables 3 and 4). This is affected by the overprediction of the flame temperature due to the assumption of adiabatic walls, as discussed in the previous section. Overall, a fair agreement of the predictions with experiments can be observed (Tables 3 and 4).

\section{Conclusions}

Turbulent flames in a generic swirl gas turbine combustor designed to operate in premixed/partially premixed mode are investigated numerically. Turbulence is modelled by a URANS formulation, using the SST turbulence model, as the basic modelling approach. For comparison, URANS is applied also in combination with the RSM to one of the investigated cases. For this case, LES is also used for turbulence modelling. For modelling the turbulence-chemistry interaction, a laminar flamelet model based on the mixture fraction and reaction progress variable is used, coupled with a presumed probability density function approach. Natural gas and syngas flames with and without external flue gas recirculation are investigated. Comparing the predictive performances of different turbulence models for one of the cases (NG-FGR20), it was observed that LES predicts a slightly thicker flame brush compared to URANS-SST, while URANS-RSM predicts a slightly sharper and more inclined flame front compared to URANS-SST, extending deeper into the burner on the axis. The numerical results obtained by different turbulence models are observed to show a comparable overall performance and a fair overall agreement with the experimental data. The slight overprediction of the combustor exit temperature is assumed to be affected by the assumption of adiabatic walls in the mathematical model. The model will be improved in the future to include nonadiabatic effects, which is also expected to lead to a more accurate prediction of NO emissions. For syngas, an increased flashback propensity could be predicted, which qualitatively agrees with the experimental observations.

\section{Nomenclature}

C: Reaction progress variable $(-)$

$c_{p}$ : Mean specific heat capacity at constant pressure $\left(\mathrm{J} \mathrm{kg}^{-1} \mathrm{~K}^{-1}\right.$ )

$k$ : Turbulence kinetic energy $\left(\mathrm{m}^{2} \mathrm{~s}^{-2}\right)$

$l_{K}$ : Kolmogorov length scale $(\mathrm{m})$

p: Static pressure $(\mathrm{Pa})$

$P$ : Favre or Reynolds probability density function (-)

Q: Volumetric heat release rate $\left(\mathrm{J} \mathrm{m}^{-3} \mathrm{~s}^{-1}\right)$

$S_{j}$ : Source term of transport equation for species $j$ $\left(\mathrm{kg} \mathrm{m}^{-3} \mathrm{~s}^{-1}\right)$

$t$ : Time (s)

T: $\quad$ Static temperature $(\mathrm{K})$

$x_{i}$ : Space coordinates $(\mathrm{m})$

$u_{j}$ : Velocity vector $\left(\mathrm{ms}^{-1}\right)$

$U_{x}$ : Axial velocity $\left(\mathrm{ms}^{-1}\right)$

$U$ : Velocity magnitude $\left(\mathrm{ms}^{-1}\right)$

$Y_{j}:$ Mass fraction of species $j(-)$

$y^{+}:$Dimensionless wall distance $(-)$

$Z$ : Mixture fraction (-).

\section{Greek Symbols}

$\beta$ : Beta probability density function (Favre or Reynolds) $(-)$

$\Delta:$ Local finite volume cell size $(\mathrm{m})$

$\varepsilon:$ Dissipation rate of $k\left(\mathrm{~m}^{-2} \mathrm{~s}^{-3}\right)$

$\theta_{C}$ : Source term for progress variable equation $\left(\mathrm{kg} \mathrm{m}^{-3} \mathrm{~s}^{-1}\right)$

$\mu$ : Viscosity $\left(\mathrm{kg} \mathrm{m}^{-1} \mathrm{~s}^{-1}\right)$

$\rho:$ Density $\left(\mathrm{kg} \mathrm{m}^{-3}\right)$

$\sigma:$ Schmidt number (-)

$\varphi$ : Thermochemical variable to be extracted from flamelet libraries (-)

$\chi$ : Scalar dissipation rate $\left(\mathrm{s}^{-1}\right)$

$\omega$ : Turbulence eddy frequency $\left(\mathrm{s}^{-1}\right)$. 


$$
\begin{aligned}
& \text { Superscripts } \\
& \text { 〜: Favre-averaged value } \\
& \text {-: Reynolds-averaged value } \\
& \text { II: Favre fluctuational value. }
\end{aligned}
$$

\author{
Subscripts \\ $b$ : Burnt \\ st: Stoichiometric \\ $t$ : Turbulent \\ $u$ : Unburnt
}

\section{Abbreviations}

EDC: Eddy dissipation concept

FGR: Flue gas recirculation

GI: $\quad$ Grid Index

GT: Gas turbine

GTC: Gas turbine combustion/combustor

LES: Large Eddy Simulation

LFM: Laminar flamelet method

NG: $\quad$ Natural gas

PDF: Probability density function

RANS: Reynolds Averaged Numerical Simulation

RMS: $\quad$ Root Mean Square

RSM: Reynolds Stress Model

SG: $\quad$ Syngas

URANS: Unsteady RANS

SST: $\quad$ Shear Stress Transport.

\section{Competing Interests}

The authors declare that they have no competing interests.

\section{References}

[1] C. Fureby, "Large eddy simulation modelling of combustion for propulsion applications," Philosophical Transactions of the Royal Society A: Mathematical, Physical and Engineering Sciences, vol. 367, no. 1899, pp. 2957-2969, 2009.

[2] D. Lörstad, A. Lindholm, N. Aklin et al., "Experimental and LES investigation of a SGT-800 burner in a combustion rig," in Proceedings of the ASME Turbo Expo 2010: Power for Land, Sea, and Air, Paper no GT2010-22688, Glasgow, UK, June 2010.

[3] G. Goldin, F. Montana, and S. Patil, "A comparison of RANS and LES of an industrial lean premixed burner," in Proceedings of the ASME Turbo Expo 2014: Turbine Technical Conference and Exposition, Paper No GT2014-25352, Düsseldorf, Germany, June 2014.

[4] R. Kulkarni, B. Bunkute, F. Biagioli, M. Duesing, and W. Polifke, "Large eddy simulation of ALSTOMs reheat combustor using tabulated chemistry and stochastic-fields combustion model," in Proceedings of the Turbo Expo 2014: Turbine Technical Conference and Exposition, Paper no GT2014- 26053, Düsseldorf, Germany, June 2014.

[5] A. C. Benim, A. Nahavandi, and K. J. Syed, "URANS and LES analysis of turbulent swirling flows," Progress in Computational Fluid Dynamics, vol. 5, no. 8, pp. 444-454, 2005.

[6] A. C. Benim, M. P. Escudier, A. Nahavandi, A. K. Nickson, K. J. Syed, and F. Joos, "Experimental and numerical investigation of isothermal flow in an idealized swirl combustor," International Journal of Numerical Methods for Heat \& Fluid Flow, vol. 20, no. 3, pp. 348-370, 2010.

[7] A. C. Benim, S. Iqbal, A. Nahavandi, W. Meier, A. Wiedermann, and F. Joos, "Analysis of turbulent swirling flows in an isothermal gas turbine combustor model," in Proceedings of the ASME Turbo Expo 2014: Turbine Technical Conference and Exposition, Paper No GT2014-25008, Düsseldorf, Germany, June 2014.

[8] B. F. Magnussen, "On the structure of turbulence and generalized eddy dissipation concept for chemical reactions in turbulent flow," in Proceedings of the 19th Aerospace Sciences Meeting, Aerospace Sciences Meetings, pp. 1-6, St. Louis, Mo, USA, January 1981.

[9] N. Peters, Turbulent Combustion, Cambridge University Press, Cambridge, UK, 2002.

[10] T. Poinsot, D. Veynante, and S. Candel, "Diagrams of premixed turbulent combustion based on direct simulation," Symposium (International) on Combustion, vol. 23, no. 1, pp. 613-619, 1991.

[11] A. C. Benim and K. J. Syed, "Laminar flamelet modelling of turbulent premixed combustion," Applied Mathematical Modelling, vol. 22, no. 1-2, pp. 113-136, 1998.

[12] S. Fischer, D. Kluss, and F. Joos, "Experimental investigation of a fuel flexible generic gas turbine combustor with external flue gas recirculation," in Proceedings of the ASME Turbo Expo 2014: Turbine Technical Conference and Exposition, Paper No GT201425388, Düsseldorf, Germany, June 2014.

[13] http://www.openfoam.com/.

[14] B. E. Launder, G. J. Reece, and W. Rodi, "Progress in the development of a Reynolds-stress turbulent closure," Journal of Fluid Mechanics, vol. 68, no. 3, pp. 537-566, 1975.

[15] M. Lesieur, O. Métais, and P. Comte, Large-Eddy Simulations of Turbulence, Cambridge University Press, New York, NY, USA, 2005.

[16] T. Poinsot and D. Veynante, Theoretical and Numerical Combustion, R. T. Edwards Inc., Philadelphia, Pa, USA, 2nd edition, 2005.

[17] C. D. Pierce and P. Moin, "Progress-variable approach for large-eddy simulation of non-premixed turbulent combustion," Journal of Fluid Mechanics, vol. 504, pp. 73-97, 2004.

[18] K. N. C. Bray, "The interaction between turbulence and combustion," in Proceedings of the 17th International Symposium on Combustion, pp. 223-233, The Combustion Institute, Pittsburgh, Pa, USA, 1978.

[19] H. Pitsch, "A C++ computer program for 0-D and 1-D laminar flamelet calculations," Tech. Rep., RWTH, Aachen, Germany, 1998.

[20] http://combustion.berkeley.edu/gri-mech/.

[21] I. B. Celik, Z. N. Cehreli, and I. Yavuz, "Index of resolution quality for large eddy simulations," Journal of Fluids Engineering, vol. 127, no. 5, pp. 949-958, 2005.

[22] A. C. Benim and K. J. Syed, Flashback Mechanisms in Lean Premixed Gas Turbine Combustion, Academic Press, New York, NY, USA, 2015. 


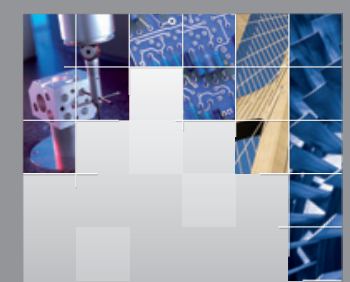

\section{Enfincering}
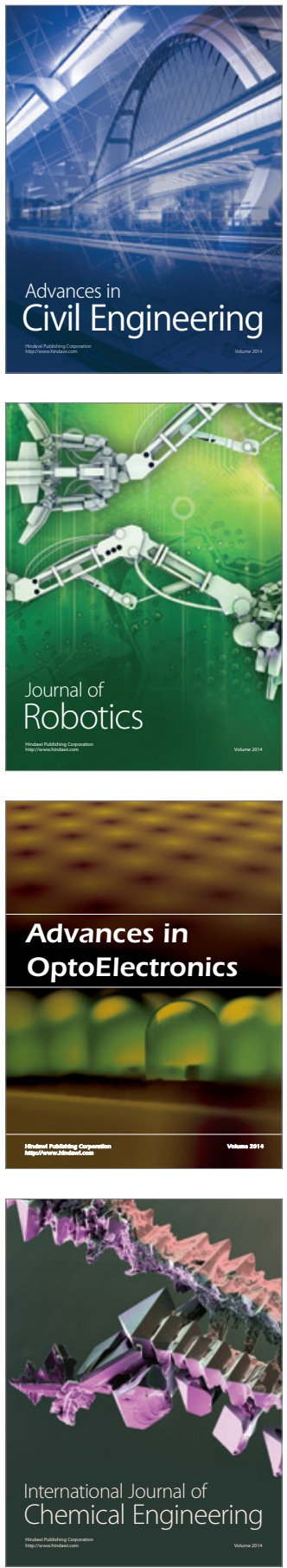

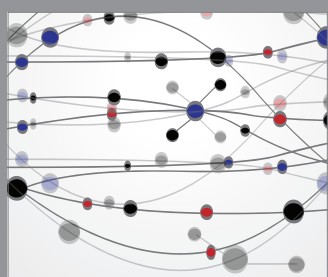

The Scientific World Journal

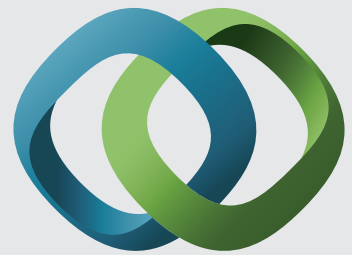

\section{Hindawi}

Submit your manuscripts at

http://www.hindawi.com
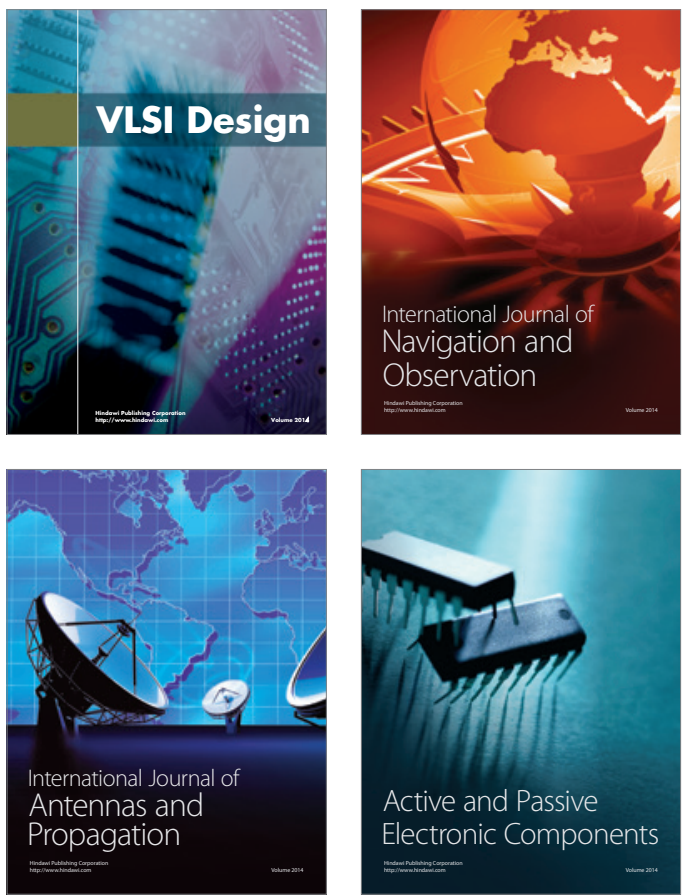
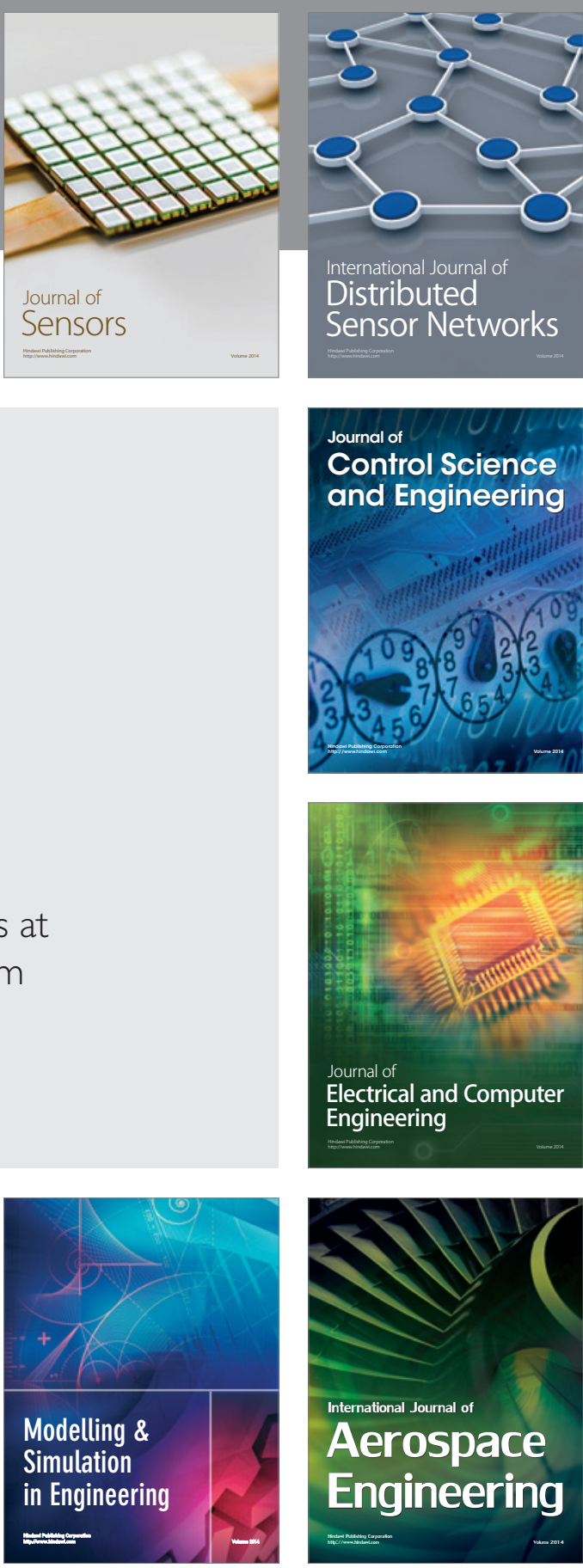

International Journal of

Distributed

Sensor Networks

Journal of

Control Science

and Engineering
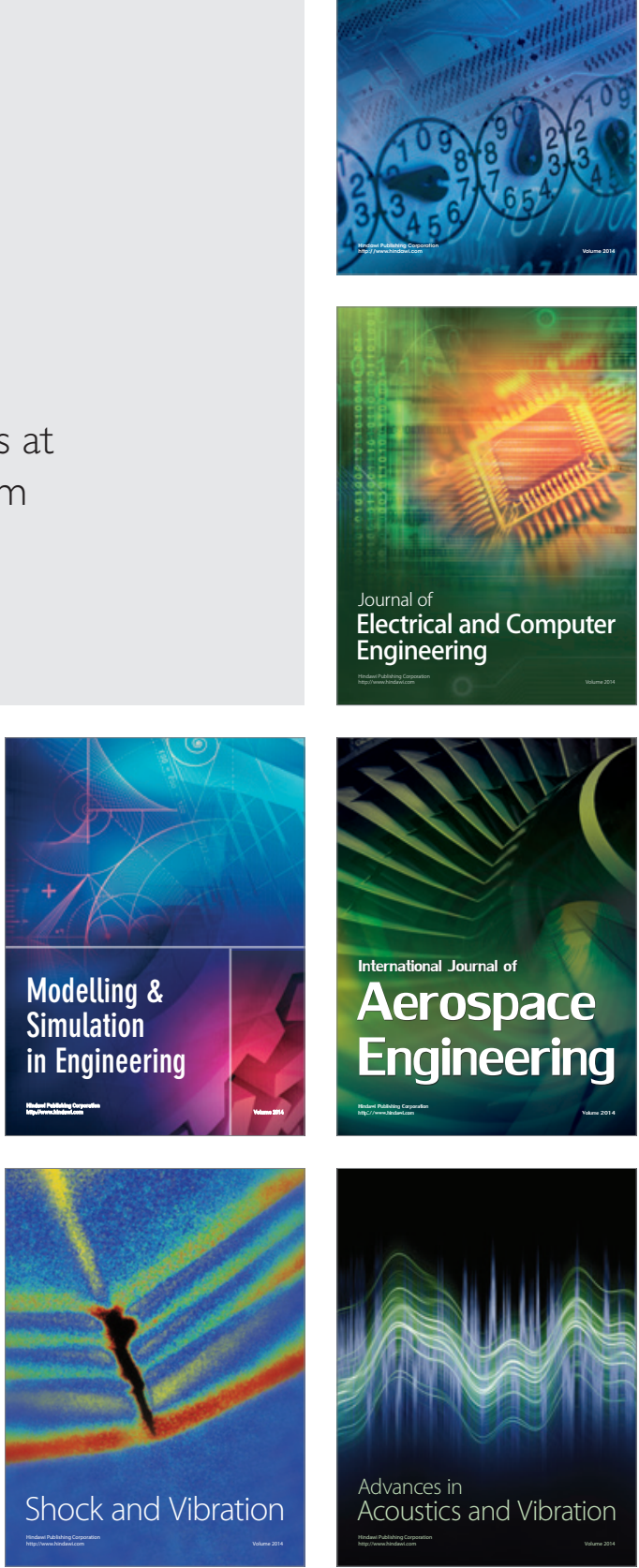